\title{
Monte-Carlo simulation study of the two-stage percolation transition in enhanced binary trees
}

\author{
Tomoaki Nogawa ${ }^{1}$ and Takehisa Hasegawa ${ }^{2}$ \\ ${ }^{1}$ Department of Applied Physics, The University of Tokyo, 7-3-1 Hongo, Bunkyo-ku, \\ Tokyo 113-8656, Japan \\ 2 Department of Physics, Hokkaido University, Kita-ku Kita 10-chome Nishi 8-chome, \\ Sapporo, Hokkaido 060-0810, Japan \\ E-mail: nogawa@serow.t.u-tokyo.ac.jp
}

\begin{abstract}
We perform Monte-Carlo simulations to study the Bernoulli $(p)$ bond percolation on the enhanced binary tree which belongs to the class of nonamenable graphs with one end. Our numerical results show that the system has two distinct percolation thresholds $p_{c 1}$ and $p_{c 2}$. The mean cluster size diverges as $p$ approaching to $p_{c 1}$ from below. The system is critical at all the points in the intermediate phase $\left(p_{c 1}<p<p_{c 2}\right)$ and there exist infinitely many infinite clusters. In this phase the corresponding fractal exponent continuously increases with $p$ from zero to unity. Above $p_{c 2}$ the system has a unique infinite cluster.
\end{abstract}

PACS numbers: 64.60.ah, 68.35.Rh, 64.60.al, 89.75.Hc 

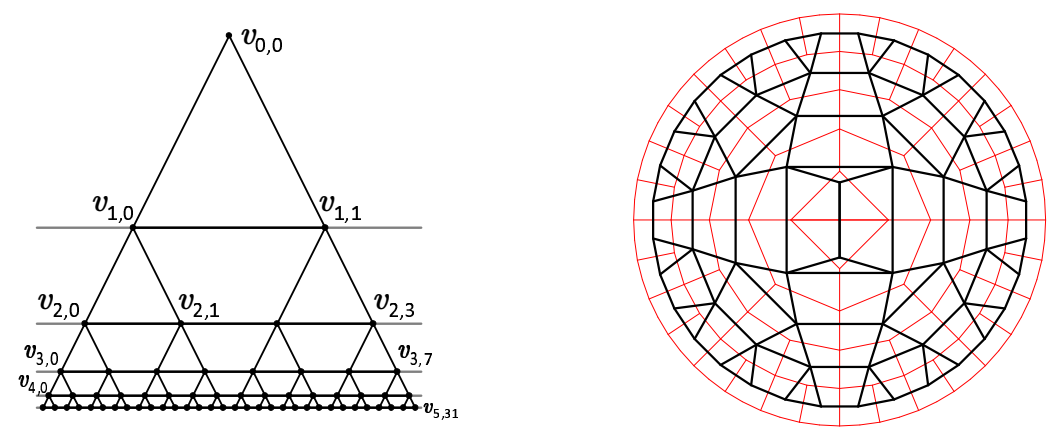

Figure 1. (left) Schematic diagram of the enhanced binary tree with 6 generations. The dangling bond on left and right side is connected in the periodic boundary condition. (right) Another presentation of EBT (thick-black line) and its dual lattice (thin-red line). For easiness to see, the root vertex is not displayed for both the EBT and the dual lattice.

\section{Introduction}

Geometry of a space, which is characterized by dimensionality and topology, is a very important factor for the collective dynamics and statics of the elements embedded on it. It takes special significance in critical phenomena where correlation length diverges. Critical phenomena have been one of the main subjects of statistical physics and intensively studied over various systems such as interacting spin models, map cellularautomata, percolation, etc. In recent years, more and more attention has been paid to critical phenomena on a class of graphs which have quite different properties with those on the well-studied Euclidean lattices. One representative example is the class, so-called blue(almost) transitive nonamenable graphs (NAGs) [1, 2], e.g., Cayley trees, hyperbolic lattices and enhanced trees [3] (see figure 1 for the binary case). Here "transitive" graph means that all vertices on it play exactly the same role in the large size limit. The fundamental property of transitive NAGs is that the number of vertices in a ball centered at the origin increases exponentially with the radius. Recent studies have reported that this property crucially affects the collective dynamics to make it differ much from those on Euclidean lattices [1, 2, 4, 5, 6, 7, 8, 9, 3, 10, Although it seems that such graphs are non-realistic and studies on them are only for theoretical interests, their distinctive features, so-called small-world property and a non-zero clustering coefficient, are common with complex networks, which are widely found in the social and natural systems [11, 12]. For example, percolation problems on complex networks are related to error and attack tolerance and cascade failures of real world systems [13]. Instead of extensive studies the interplays between network structures and critical phenomena is still unclear. Transitive NAGs has large advantages in its simplicity, e.g., regularity of the lattice, compared with usually studied complex networks. Studies on them has have a possibility to give a breakthrough on this complex problem.

The number of ends, which indicates a kind of vulnerability of graphs, is an important concept for NAGs. The number of ends $e(G)$ of an infinite graph $G$ is 
defined as the supremum of the number of infinite connected components in $G \backslash S$, where $G \backslash S$ is the graph obtained from $G$ by removing arbitrary finite subgraph $S$ and the bonds incident to those. While trees have infinitely many ends, hyperbolic lattices and enhanced trees have only one end. Recent analytical studies have indicated that the Bernoulli bond percolation on NAGs with one end exhibits a new type of phase transition, so-called multiple phase transition (MPT), which takes three distinct phases according to open bond probability $p$ as follows [1, 2]:

- Nonpercolating phase: there is no infinite cluster for $0 \leq p<p_{c 1}(G)$.

- Intermediate phase: there are infinitely many infinite clusters for $p_{c 1}(G)<p<$ $p_{c 2}(G)$.

- Percolating phase: there is a unique infinite cluster for $p_{c 2}(G)<p \leq 1$.

Here infinite cluster means a cluster whose mass diverges with system size $N$ as $N^{\psi}$ with $0<\psi \leq 1$. As well known for the Euclidean lattices, any amenable graph, e.g., can have at most only one infinite cluster, i.e., $p_{c 1}=p_{c 2}$ [14]. By contrast there are always plural infinite clusters in the percolating phases of trees. This is a consequence of infinitely many ends, i.e., trees have no long cyclic paths and split easily. Both of an exponential growth and sufficient amount of loops are necessary for graphs to exhibit three distinct phases. Indeed, Benjamini and Schramm proved the existence of a MPT on any planar transitive NAG with one end [10]. In addition, it is proved that the transition at $p_{c 1}$ belongs to the mean-field universality class [15].

Previous studies of MPTs have been restricted in the analytic way based on probability theory and we lack numerical research, which will bring us the quantitative indication of MPTs. Especially finite size effect is hardly investigated by the analytic approach. This effect is crrucial to argue the NAGs and the realistic network, where the typical length scale of the graphs often grows as a logarithmic function of the total number of vertices $N$ and is still small for considerably large $N$. In this paper, we investigate a MPT by Monte-Carlo simulations taking the enhanced binary tree (EBT) as an example. Our numerical results actually give two distinct critical probabilities $p_{c 1}$ and $p_{c 2}$. All the points in the intermediate phase $\left(p_{c 1}<p<p_{c 2}\right)$ are critical and there exist infinitely many infinite clusters . The corresponding fractal exponent continuously increases with $p$ from 0 to 1 and bridges a gap between non-percolating and percolating phases.

\section{Fundermental mechanism of the two-stage transition}

In this section we provide a basic picture of MPTs on NAGs. Although we consider the EBT as an example, the concept is not specified to it.

At first we define an enhanced binary tree with $L$ generations. We prepare vertices $v_{n, m}$ for $n=0,1, \cdots, L-1$ and $m=0,1, \cdots, 2^{n}-1$. The total number of vertices $N$ equals $2^{L}-1$, i.e., $L \approx \log _{2} N$. Inter-generation (radial) bonds are supposed between $v_{n, m}$ and $v_{n+1,2 m}$ and between $v_{n, m}$ and $v_{n+1,2 m+1}$. These bonds yield a binary 
tree. Furthermore intra-generation (circumferential) bonds are added between $v_{n, m}$ and $v_{n, m+1}$, which means "enhanced". All of inter- and intra-generation bonds are open with homogeneous probability $p$ to yield connected clusters. EBT is not exactly transitive but almost transitive since the root vertex $v_{0,0}$ is special and the center can be defined. But this presumably does not affect the essential property of the critical phenomena.

The following scenario is based on the natural assumption that the connectedness (correlation) function; the probability that a vertex of the $\ell$-th generation is connected to the root clster, that $v_{0,0}$ belongs to, exponentially decays wiht $\ell$ as

$$
C_{0}(\ell) \propto 2^{-\ell / \xi}
$$

Here $\xi$ means a correlation length along the generation (radial) direction and it monotonically increases with bond opening probability $p$. The averaged mass of the root cluster $\left\langle s_{0}\right\rangle$ is calculated by summing up the connectedness function as

$$
\left\langle s_{0}\right\rangle \propto \sum_{\ell=0}^{L-1} 2^{\ell} C_{0}(\ell)=\frac{\alpha^{L}-1}{\alpha-1} \quad \text { where } \quad \alpha \equiv 2^{1-1 / \xi}
$$

and $2^{\ell}$ is the number of vertices at the $\ell$-th generation taking a role of surface area factor. For $\xi<1$ and $\alpha<1,\left\langle s_{0}\right\rangle$ converges to finite value in the thermodynamic limit $L \rightarrow \infty$. On the other hand $\left\langle s_{0}\right\rangle$ diverges with $L$ for $\xi \geq 1$ and $\alpha \geq 1$. It is important that the mass of cluster diverges despite of finite correlation length owing to the exponentially diverging surface area. This is an exact mechanism for the percolation transition on the binary Cayley tree, where $C_{0}(\ell)=p^{\ell}$ and $\xi=1 / \log _{2}(1 / p)$. We can define the critical probability $p_{c 1}$ at which a correlation mass diverges. From equation (2), $\left\langle s_{0}\right\rangle$ diverges very slowly with respect to the system size as

$$
\left\langle s_{0}\right\rangle_{L} \propto L \approx \log _{2} N \quad \text { at } \quad p=p_{c 1} .
$$

Above $p_{c 1},\left\langle s_{0}\right\rangle$ diverges with the system size as

$$
\left\langle s_{0}\right\rangle_{L} \propto \alpha^{L}=2^{L \log _{2} \alpha}=N^{\psi} .
$$

where

$$
\psi \equiv \log _{2} \alpha=1-1 / \xi
$$

The exponent $\psi$ mimics $d_{f} / d$ where $d_{f}$ is the fractal dimension of a cluster embedded on the $d$-dimensional Euclidean lattice. Note that $\psi$ is a function of $\xi$ and increases with $p$. Even though a cluster mass diverges above $p_{c 1}$, the ratio $\left\langle s_{0}\right\rangle / N$, which can be regarded as an order parameter, goes to zero for $N \rightarrow \infty$ as far as $\psi<1$. If $\xi$ diverges at a certain $p, \psi$ continuously approaches to 1 . We define the critical probability $p_{c 2}$ at which the correlation length diverges. At this critical point a prefactor of the connectedness function in equation (11) in form $\ell^{-\tilde{\eta}}$ takes an important role. The exponent $\tilde{\eta}$ corresponds to $2-d+\eta$ for the $d$-dimensional Euclidean lattice. Positive $\tilde{\eta}$ makes $\left\langle s_{0}\right\rangle / N$ vanish in the thermodynamic limit and the order parameter continuously rising from zero at $p_{c 2}$. In this case, however, $\left\langle s_{0}\right\rangle / N$ at $p_{c 2}$ approaches to zero very slowly with $N$ as $\left(\log _{2} N\right)^{-\tilde{\eta}}$. 
The finite size dependences are summarized as

$$
\left\langle s_{0}\right\rangle \propto\left\{\begin{array}{ccl}
N^{0} & \text { for } & p<p_{c 1} \\
\log _{2} N & \text { at } & p=p_{c 1} \\
N^{\psi(p)} & \text { for } & p_{c 1}<p<p_{c 2} \\
N /\left(\log _{2} N\right)^{-\tilde{\eta}} & \text { at } & p=p_{c 2} \\
N & \text { for } & p>p_{c 2} .
\end{array}\right.
$$

The cluster mass shows fractal behavior in the phase $p_{c 1}<p<p_{c 2}$ in contrast with the Euclidean lattice which has a critical point and unique fractal exponent. We consider the discrepancy of the probabilities for correlation mass and length is the key concept of the MPT on NAGs, which never occurs on the Euclidean lattices. In the following section we confirm the validity of the scenario shown above by numerical simulations.

\section{Numerical results}

Here let us explain the detail of our Monte-Carlo simulations. The systems with $L=10-22$ are investigated. We generate samples, i.e., sets of open and closed bonds, by using pseudo random number generated by the Mersenne-Twister method [16]. The ensemble average of observed quantities is taken over 480,000 samples. In order to improve the precision of the average values for small $p$, we treat the bonds which is connected to initial 3 generations exactly, i.e., counting all $2^{3 \times\left(2^{3}-1\right)-2}$ possible realizations with probability $p^{n_{o}}(1-p)^{n_{c}}$, where $n_{o}\left(n_{c}\right)$ is the number of open (closed) bonds. This treatment only takes the CPU-time independent of $N$.

We also investigate the percolation on the dual lattice of the EBT (see figure 11), which is also a NAG with one end. Each vertex of the dual lattice is put on the center of triangle or rectangle cells of the EBT and each bond, which is open with probability $\bar{p}$, crosses with the conjugate bond of the EBT. A duality relation

$$
p_{c 1}+\bar{p}_{c 2}=1 \quad \text { and } \quad p_{c 2}+\bar{p}_{c 1}=1
$$

is known for dual planar NAGs [10].

As mentioned in the previous section the $N$-dependence of $\left\langle s_{0}\right\rangle$ for the finite size systems determines the expected three phases. So we estimate a fractal exponent

$$
\psi=\frac{d \ln \left\langle s_{0}\right\rangle_{N}}{d \ln N} \approx \frac{d}{d L} \log _{2}\left\langle s_{0}\right\rangle_{N},
$$

so that $\left\langle s_{0}\right\rangle \propto N^{\psi}$. In practice, we calculate $\psi$ by the difference $\left[\ln \left\langle s_{0}\right\rangle_{2 N}-\right.$ $\left.\left.\ln \left\langle s_{0}\right\rangle_{N / 2}\right)\right] /[\ln (2 N)-\ln (N / 2)]$. This is a good approximation of equation (8) for large $N$ when $\left\langle s_{0}\right\rangle$ is a power function of $N$. The $p$-dependences of $\psi$ for both of the EBT and the dual lattice are shown in figure 2. Three distinct phases can be observed as expected. The exponent $\psi$ of the EBT grows from zero to unity in the intermediate phase between $p \approx 0.30$ and $p \approx 0.56$. Oppositely $\psi$ of the duality lattice decrease from unity to zero between $1-\bar{p} \approx 0.30$ and $1-\bar{p} \approx 0.56$. This suggests the duality relation actually holds. 


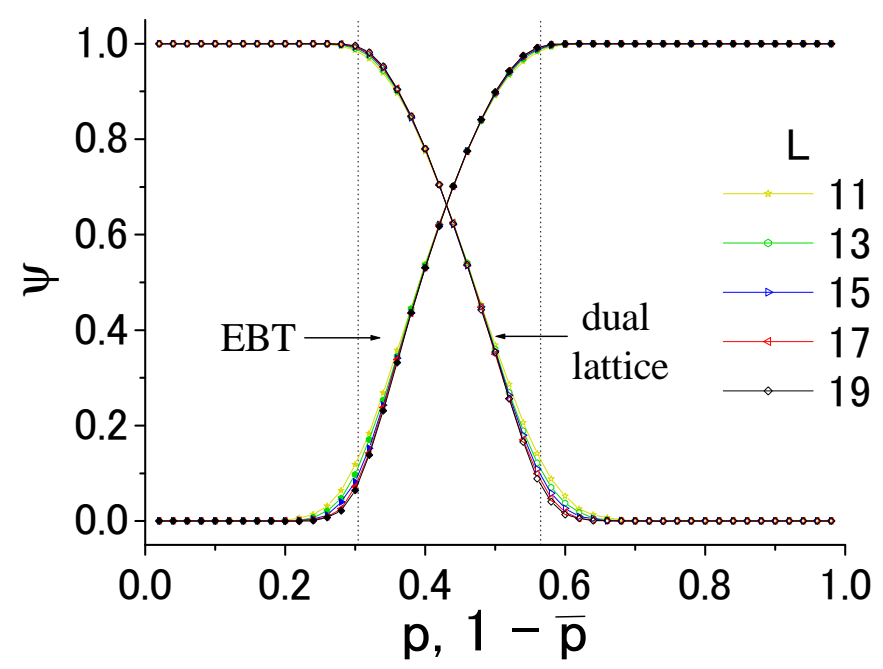

Figure 2. The $p$-dependence of $\psi$ for the enhanced binary tree and its dual lattice. For the latter, the horizontal axis is $1-\bar{p}$ to cofirm the duality relation. Results for various $L$ 's are shown together. The two vertical lines indicates $p=0.304$ and $p=0.563$, respectively.

The $N$-dependence of $\left\langle s_{0}\right\rangle$ at $p_{c 1}$ is decided as follows. Approaching $p_{c 1}$ from above, one expects that $\psi$ goes to zero but $\left\langle s_{0}\right\rangle$ diverges as $\ln N(\propto L)$ at $p=p_{c 1}$. To exclude the possibility that $\left\langle s_{0}\right\rangle \propto L^{\varphi}$ with $\varphi \neq 1$, we plot

$$
\varphi_{L}(p)=\frac{d \ln \left\langle s_{0}\right\rangle_{N}}{d \ln L}
$$

as a function of $p$ in figure 3. As $L$ goes to infinity, $\varphi_{L}(p)$ decreases to zero for $p<p_{c 1}$ and diverges for $p>p_{c 1}$. Only at $p=p_{c 1}, \varphi_{L}$ converges to $N$-independent value, which is estimated at unity. Practically we obtain the precise critical probability $p_{c 1}=0.304(1)$ from this crossing point and $\bar{p}_{c 1}=0.436(1)$ in the same way. Using the duality relation (7), $p_{c 2}=0.564(1)$ and $\bar{p}_{c 1}=0.696(1)$ are also determined.

In figure 4 we show the geometry of the root cluster on the EBT by choosing samples which has a relatively large size cluster. Figure 4(a) is for $p=0.304 \approx p_{c 1}$. The cluster survive marginally; its branches do not show spreading behavior, and therefore the mass of the cluster is proportional to the number of generations. Figure 4(b) shows the cluster at a middle point in the intermediate phase, which has many branches. The branches are spreading with the generation and tend to diverge. However this cluster occupies very small part of the whole system because spreading rate is slower than that of the EBT itself. Consequently, there is a room for other infinitely large clusters. Figure 4 (c) shows the cluster at $p=0.564 \approx p_{c 2}$. It looks compact and will occupy the finite fraction of the whole system in the thermodynamic limit.

Next we estimate the critical exponents of the first transition at $p=p_{c 1}$. Let us suppose a finite size scaling law

$$
\left\langle s_{0}\right\rangle \propto L^{\gamma \bar{\nu} / \nu} \tilde{s}_{0}\left(\left(p_{c 1}-p\right) L^{\bar{\nu} / \nu}\right),
$$



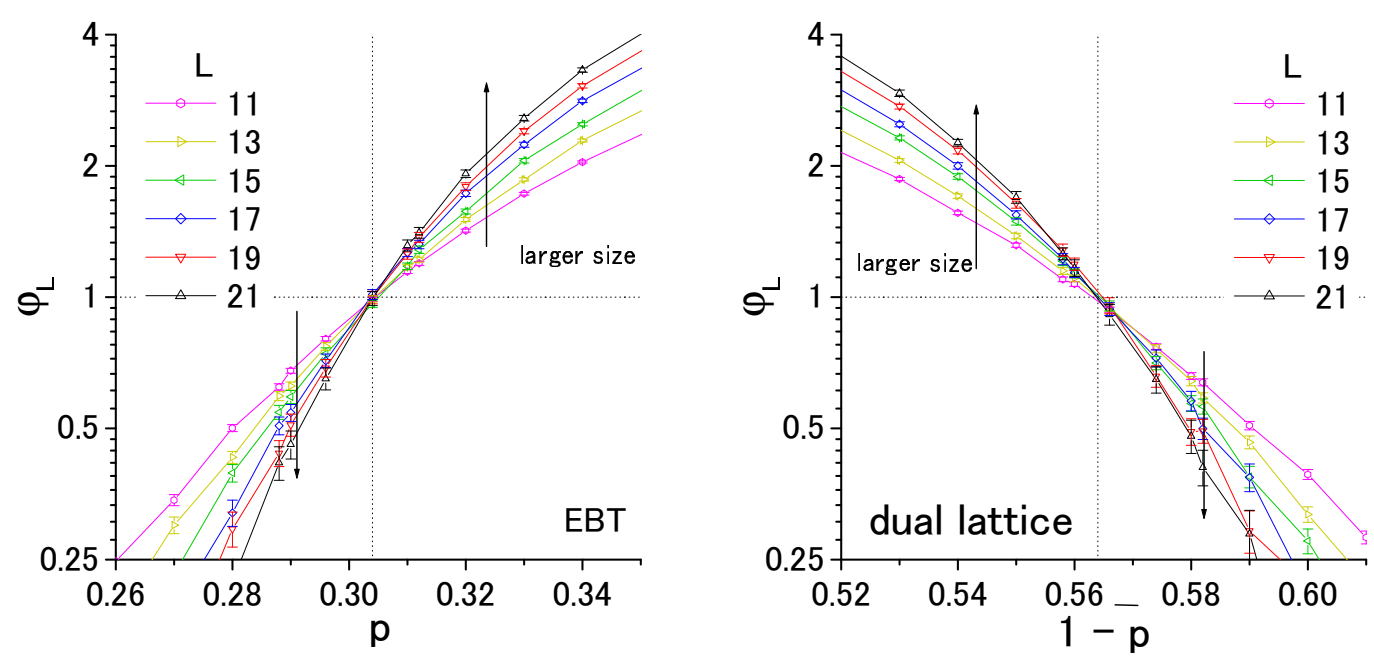

Figure 3. The $p$-dependence of $\varphi_{L}$ for the EBT (left) and its dual lattice (right) with several system sizes. The crossing point indicates the transition point where $\left\langle s_{0}\right\rangle$ logarithmically diverges.

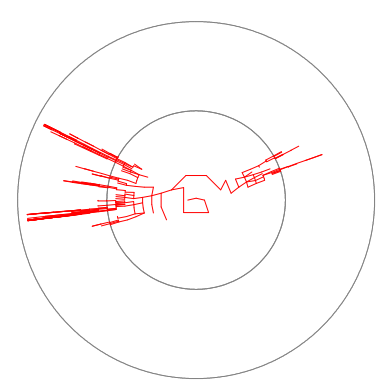

(a)

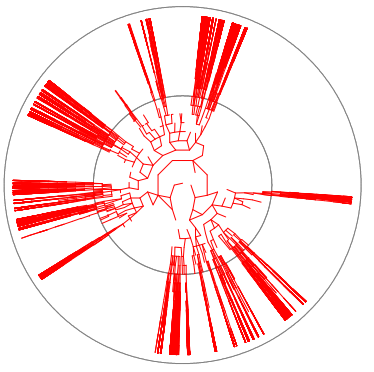

(b)

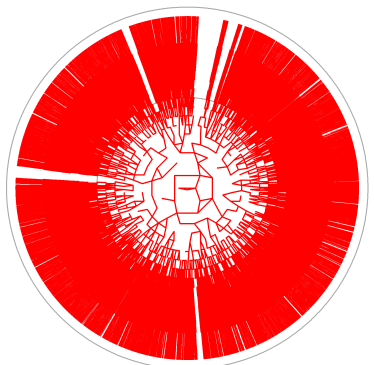

(c)

Figure 4. Typical geometry of giant clusters in the system with $L=20$ for $p=0.304$ (a), $0.400(\mathrm{~b}), 0.564(\mathrm{c})$.

for $p<p_{c 1}$. $甘$ The scaling function $\tilde{s}_{0}(\cdot)$ should have asymptotic forms,

$$
\tilde{s}_{0}(x) \propto\left\{\begin{array}{cl}
\text { const. } & \text { for } x \ll 1 \\
x^{-\gamma} & \text { for } x \gg 1
\end{array}\right.
$$

Figure 5 shows a nice collapsing of data by using $\gamma=1.0$ and $\bar{\nu} / \nu=1.0$. The data for the dual lattice also shows same scaling behavior (the form of scaling function is very similar to the one for the EBT). This result confirms that the first transition at $p_{c 1}$ belongs to the mean field universality class $(\gamma=1$ and $\bar{\nu}=\nu=1 / 2$ [17]) as predicted in Ref. [15]. On the other hand the transition at $p_{c 2}$ does not look like an usual continuous phase transition. The order parameter $\left\langle s_{0}\right\rangle / N$ is fitted well by $\left\langle s_{0}\right\rangle / N=0.49+0.58 L^{-0.083}$ at $p=0.564 \approx p_{c 2}$ using data for $L=10-24$ (not shown here). This means $\tilde{\eta}=0$ $\left\langle s_{0}\right\rangle / N$ has a finite limit value in $L \rightarrow \infty$ at the critical point. We have to be careful to

$\ddagger$ Consult Ref. [17 about the exponent $\bar{\nu}$. We use generation $L$ as a characteristic length instead of chemical distance in these papers. The number of retrenched horizontal paths is so small compared with vertical ones that we regard this change to be irrelevant. 


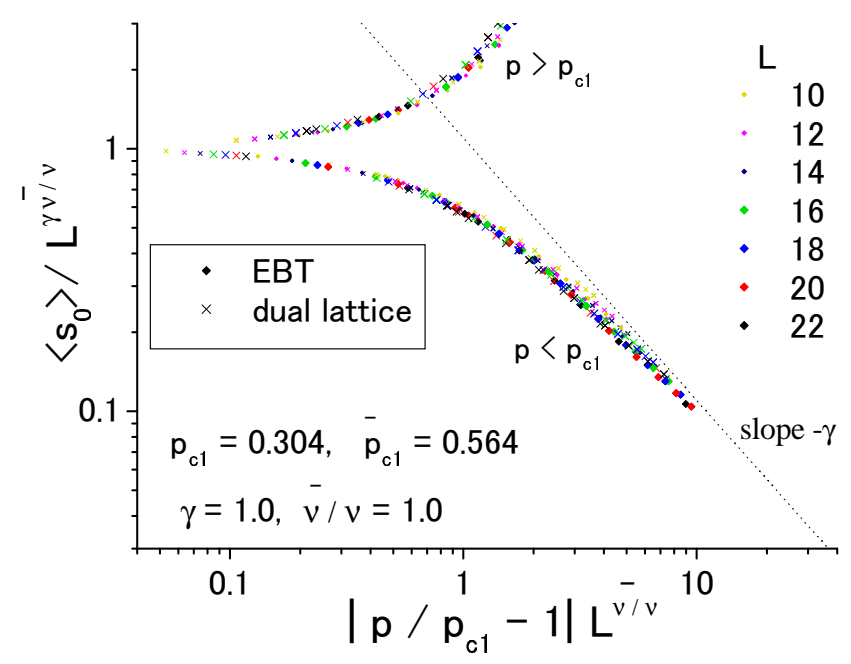

Figure 5. Finite size scaling plot of $\left\langle s_{0}\right\rangle$ as a function of $p-p_{c 1}$. The two groups, the EBT and the dual lattice, are plotted in arbitrary unit to collapse.

conclude such special feature, discontinuous transition in the framework of second order transition, but can say, at least, that $\tilde{\eta}$ is smaller than 0.083 .

Finally we investigate the distribution function $n_{s}$, which is the number of clusters with size $s$ per vertex, in order to show that the system is always critical in the intermediate phase as already implied from the $N$-dependence of $\left\langle s_{0}\right\rangle$. We assume a finite size scaling law

$$
n_{s}(N)=N^{-\psi \tau} \tilde{n}\left(s N^{-\psi}\right),
$$

for fixed $p$ between $p_{c 1}$ and $p_{c 2}$. Here the scaling function $\tilde{n}(x)$ is a power function $x^{-\tau}$ for $x \ll 1$, and a rapidly decreasing function for $x \gg 1$. In the scaling plot, figure 6 , we use $\psi$ evaluated by equation (8) and the scaling relation $\tau=1+\psi^{-1}$ at each $p$ (note that this relation is based on the scaling relation $\tau=1+d_{f} / d$ on the Euclidean lattices). Figure 6 strongly supports that the above finite size scaling (12) holds in the intermediate phase. Equation (12) indicates that the characteristic size of clusters diverges with $N \rightarrow \infty$ and a power-law distribution, $n_{s}(s) \propto s^{-\tau}$, holds up to infinite $s$. When such a power-law exists, it can be said that the number of infinite clusters is infinite because one can always find $\psi^{\prime}$ satisfying $0<\psi^{\prime}<\psi$ for any positive $\psi$ with which the number

$$
N\left(s>N^{\psi^{\prime}}\right) \equiv \int_{N^{\psi^{\prime}}}^{\infty} d s N n_{s}(N) \sim N^{\left(1-\psi^{\prime} / \psi\right)},
$$

diverges in the limit $N \rightarrow \infty$.

\section{Summary and discussions}

In conclusion, we studied the bond percolation problem on the enhanced binary tree by Monte Carlo simulations for the first time. We observed the intermediate phase in addition to the nonpercolating and percolating phases where the average cluster size is 


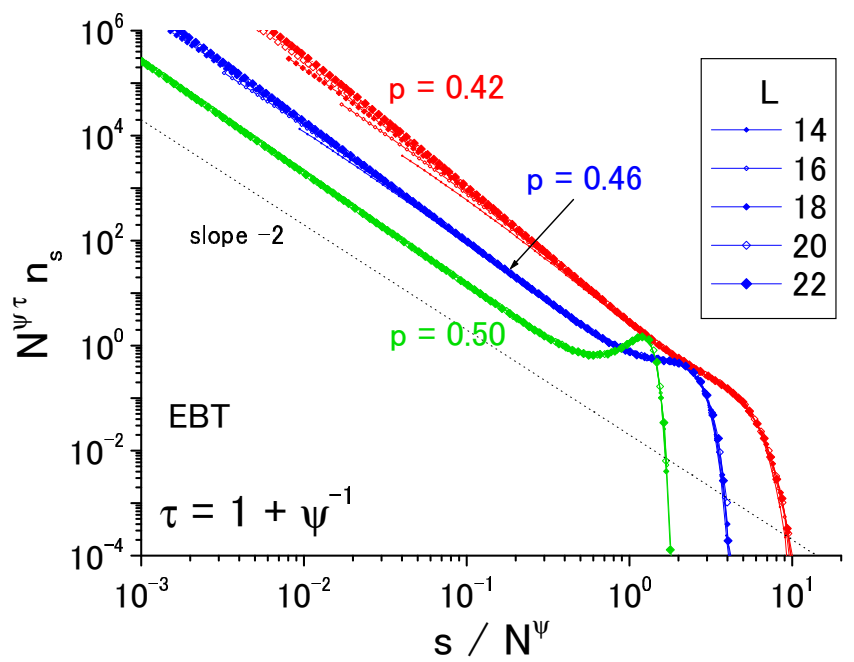

Figure 6. Finite size scaling plot of $n_{s}$ as a function of $s$ for several $p$ 's in the intermediate phase. We use the values of $\psi$ shown in figure 2. Here we eliminate the data for $(s<16)$ since the data with too small $s$ does not obey to the scaling law.

finite and in the same order of the whole system size $N$, respectively. All the points in the intermediate phase is critical and the fractal exponent $\psi$, where $\left\langle s_{0}\right\rangle \propto N^{\psi}$, increases continuously from zero to unity with increasing $p$. Note that the system looks critical in terms of mass of cluster, and the correlation length must diverge at $p_{c 2}$.

We expect that the mechanism of the MPT on the EBT is commonly observed in the other systems of NAGs with one end. There is, however, an interesting open problem, for both analytic and numerical studies, whether non-transitivity or inhomogeneity changes the scenario or not, which seriously corresponds to the complex networks.

\section{Acknowledgments}

The present work is supported by 21st Century COE program "Topological Science and Technology". We wish to thank H.Shima and Y.Sakaniwa for their stimulating works, and K.Nemoto and K.Yakubo for helpful comments and discussions.

\section{References}

[1] Lyons R 2000 J. Math. Phys. 411099

[2] Schonmann R H 2001 Commun. Math. Phys. 219271

[3] Benjamini I and Schramm O 1996 Elect. Comm. in Probab. 171

[4] Shima H and Sakaniwa Y 2006 J. Phys. A 394921

[5] Shima H and Sakaniwa Y 2006 J. Stat. Mech. P08017

[6] Ueda K, Krcmar R, Gendiar A, and Nishino T 2007 J. Phys. Soc. Jpn. 76084004

[7] Krcmar R, Gendiar A, Ueda K, and Nishino T 2008 J.Phys. A, Math. Theor. 41125001

[8] Wu C C 1996 J. Stat. Phys. 85251

[9] Wu C C 2000 J. Stat. Phys. 100893

[10] Benjamini I and Schramm O 2000 J. Amer. Math. Soc. 14487 
[11] Albert R and Barabási A -L 2002 Rev. Mod. Phys. 7447

[12] Newman M E J 2003 SIAM Review 45167

[13] Dorogovtsev S N, Goltsev A V, Mendes J F F 2008 Rev. Mod. Phys. 801275

[14] Burton R M and Keane M 1989 Commun. Math. Phys. 121501

[15] Schonmann R H 2002 Commun. Math. Phys. 225453

[16] Matsumoto M and Nishimura T 1998 ACM Trans. Model. Comput. Simul. 83

[17] Havlin S and Nossal R 1984 J. Phys. A 17427 